\title{
Telepresence Control in Packet Switched Communication Networks
}

\author{
Sandra Hirche and Martin Buss
}

\begin{abstract}
One of the crucial factors for the performance of haptic (force feedback) telepresence systems is the quality of communication. This paper investigates known telepresence control stability conditions for the application in packet switched networks with time varying communication latencies and packet loss, as e.g. the Internet, and discusses the impact of the packet processing protocol on the stability and the performance of telepresence systems with haptic feedback. In experiments - towards a multimodal telepresence system over IP with rescue and other applications - the system performance of the standard velocity/force architecture is compared with the position/velocity/force architecture.
\end{abstract}

\section{INTRODUCTION}

The goal of multimodal telepresence systems is to enable a human operator to be present and to actively perform complex manipulation tasks in possibly distant or differently scaled remote environments; see e.g. [1]. Application areas reach from telemanufacturing and telemaintenance to telesurgery and rescue applications. With the ongoing efforts to provide Quality-of-Service IP-networks become more and more attractive as communication medium for sensitive realtime applications as multimodal telepresence systems are. Realtime control of robots has been successfully performed over the Internet, first by [2] with visual feedback only, due to best-effort-characteristics of the Internet and the stability problem very few have considered haptic feedback, see [3].

A multimodal telepresence rescue system as proposed in [4] is shown in Fig. 1. The human operator manipulates the force feedback capable Human System Interface (HSI) thereby commanding the executing robot (teleoperator). While the teleoperator interacts with the usually unknown remote environment the sensor data (visual, auditory, haptic) are fed back and displayed to the operator. Ideally the human operator feels directly connected to the remote environment; the telepresence system is then transparent. Considering the visual and auditory feedback as stateof-the-art-multimedia, this paper focusses on the haptic feedback subsystem.

The sampled command signals and sensor data, both continuously generated realtime mediastreams, are communicated via a packet switched communication network, e.g. the Internet, thereby closing a global control loop.

This work was partly supported by the DFG Collaborative Research Center SFB453.

Both authors' affiliation: Institute of Automatic Control Engineering, Technische Universität München, D-80290 München, Germany

http: //www.lsr.ei.tum.de,

Sandra.Hirche@ei.tum.de_Martin.Buss@ei.tum.de

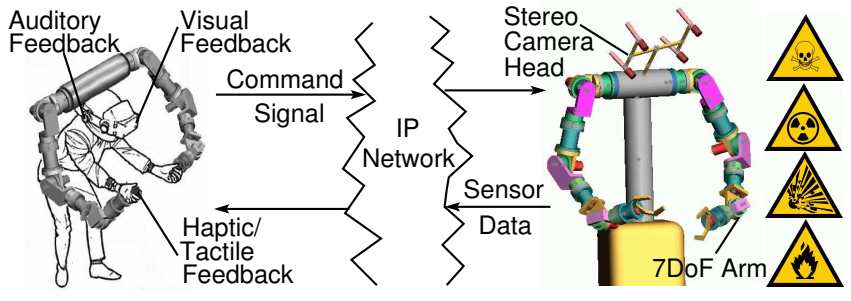

Fig. 1. Multimodal human rescuing telepresence system.

Substantial time varying delays as they occur in the Internet destabilize the closed loop system resulting in a severe hazard to the safety of the human and the remote environment.

The first breakthrough to solve this problem of haptic telepresence with time delay has been made by applying the passivity concept guaranteeing stability. The resulting control methods, as the scattering transformation and the combined velocity/force control of teleoperator/HSI, see [6], stabilize the system for arbitrary but constant delay. Recently another breakthrough has been achieved by extending the approach for time varying delays, see [7], [8]. The stability conditions though are formulated for the continuous time case, hence do not incorporate the effects of a packet switched communication network.

In such a network depending on the traffic conditions the data packets are randomly delayed, may arrive in permutated order, and packet loss may occur. The choice of the packet processing algorithm, hence the treatment of late and lost packets highly affects the performance of the telepresence system. In [9] for the time varying delay case a virtual delay is introduced through queueing, so that all data experience the worst case delay of the system. As a result the performance is degraded. Packet loss or increasing time delay may result in a buffer underrun at the receiver side. In [10] two different compensation schemes for the treatment of the resulting vacant sampling instances are proposed: keeping the old value (Hold Last Sample) or generating a new value with estimated data. Both techniques have been successfully applied in networked control systems (NCS). Packet loss compensation by holding the last received sample has also been employed in [11].

This paper investigates the stability and the performance of packet processing algorithms aiming at their application in haptic telepresenc systems communicating over packet switched IP-networks. Results stemming from the continuous time considerations as the time varying gain [7] and position/velocity/force architecture [8] are examined for their applicability in packet switched networks. In an 
experiment towards a telepresence rescue system a wall environment is haptically explored. Thereto the Cartesian $y$-position of the 7 DOF teleoperator arm, described in [12], is teleoperated by means of a 1 DOF force feedback paddle via an emulated realtime communication network, see [13].

The paper is organized as follows: section 2 introduces passivity based stabilization methods and measures of transparency; in section 3 the passivity of packet processing algorithms and the benefit of the position/velocity/force architecture is analyzed; the last two sections present the experimental system architecture and the results.

\section{THEORETICAL BACKGROUND}

The human rescuing telepresence system basically consists of a force feedback capable HSI (variables indexed ${ }_{h}$ ) and the teleoperator (index ${ }_{t}$ ) interacting with an usually unknown remote environment (index ${ }_{e}$ ) as shown in Fig. 2. In bilateral telepresence the human manipulates the HSI applying the force $f_{h}$. Based on stability arguments in the standard architecture the HSI velocity $\dot{x}_{h}$ is communicated to the teleoperator where the local velocity control loop ensures the tracking of the desired teleoperator velocity $\dot{x}_{t}^{d}$ ( ${ }^{d}$ denotes desired). The force $f_{e}$ sensed at the remote site, resulting from the interaction with the environment, is transmitted back to the HSI serving as reference signal $f_{h}^{d}$ for the local force control.

The HSI and the teleoperator are connected through a communication network closing a global control loop via the human operator. In case of a packet switched unreliable service network, as e.g. the Internet, the data packets, thus the control signals are afflicted with varying time delay and packet loss. Without further control measures the closed loop system is unstable.

\section{A. The Passivity Approach}

A common approach to analyze and synthesize telepresence system architectures with time delay is the passivity concept providing a sufficient condition for stability of the haptic telepresence system. A complex system of interconnected network elements ( $n$-ports) is passive if each of the subsystems is passive. A passive element is one for which, given zero energy storage at $t=0$, the property

$$
\int_{0}^{t} P_{\text {in }}(\tau) \mathrm{d} \tau=\int_{0}^{t} u^{T}(\tau) y(\tau) \mathrm{d} \tau \geq 0 \quad \forall t>0
$$

holds, with $P_{\text {in }}(\tau)$ denoting the power stored or dissipated in the system, $u(\tau), y(\tau)$ being the input and output vector. In classical telepresence architectures, as proposed in [6], the appropriately locally controlled HSI and teleoperator exchange velocity and force signals, as the mapping from velocity to force is generally passive, hence the teleoperator and the HSI are passive subsystems. The environment is considered passive and it is assumed that the (possibly so trained) human operator behaves in a cooperative, i.e. passive way.

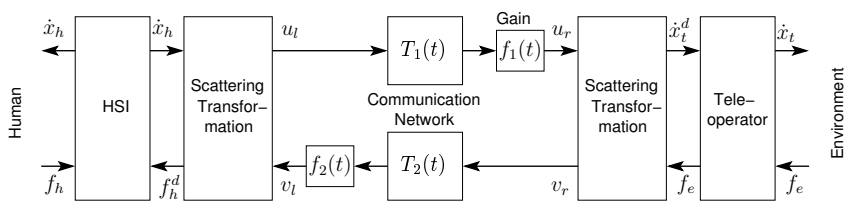

Fig. 2. System architecture for time varying delay with time varying gains $f_{1}(t), f_{2}(t)$.

In case of time delay in the communication network the bidirectional communication channel can be modeled as a time delaying two-port with time varying delays $T_{1}(t)$, $T_{2}(t)$ in the forward and the backward path, respectively, as shown in the network representation in Fig. 2. The blocks of the scattering transformation and the time varying gains $f_{1}(t), f_{2}(t)$ are explained below. Applying scattering theory it is straightforward to show that the eigenvalues of the scattering matrix are larger than 1, i.e. the passivity condition is violated; the communication line generates energy. The scattering transformation first applied to telepresence systems in [6] and extended in [14] passifies the communication two-port for constant delays $T_{1}(t)=T_{1}$, $T_{2}(t)=T_{2}$ with the wave variable transformation

$$
\begin{array}{ll}
u_{l}=\frac{1}{\sqrt{2 b}}\left(f_{h}^{d}+b \dot{x}_{h}\right) ; & u_{r}=\frac{1}{\sqrt{2 b}}\left(f_{e}+b \dot{x}_{t}^{d}\right) ; \\
v_{l}=\frac{1}{\sqrt{2 b}}\left(f_{h}^{d}-b \dot{x}_{h}\right) ; & v_{r}=\frac{1}{\sqrt{2 b}}\left(f_{e}-b \dot{x}_{t}^{d}\right) .
\end{array}
$$

Using the notation of (1) the input vector is given by $u^{T}=\left[\dot{x}_{h}-\dot{x}_{t}^{d}\right]$ and the output vector by $y^{T}=\left[f_{h}^{d} f_{e}\right]$. Disregarding the blocks $f_{1}(t), f_{2}(t)$ in Fig. 2, the communication two-port now becomes passive, i.e. does not generate energy

$$
\begin{aligned}
\int_{0}^{t} P_{i n}(\tau) \mathrm{d} \tau & =\int_{0}^{t}\left(\dot{x}_{h} f_{h}^{d}-\dot{x}_{t}^{d} f_{e}\right) \mathrm{d} \tau \\
& =\frac{1}{2} \int_{0}^{t}\left(u_{l}^{2}-u_{r}^{2}+v_{r}^{2}-v_{l}^{2}\right) \mathrm{d} \tau \\
& =\frac{1}{2} \int_{t-T_{1}}^{t} u_{l}^{2} \mathrm{~d} \tau+\frac{1}{2} \int_{t-T_{2}}^{t} v_{r}^{2} \mathrm{~d} \tau \geq 0 \quad \forall t .
\end{aligned}
$$

If the delay varies over time the passivity condition for the communication two-port/scattering transformation may be violated depending on the rate of change of the delay as shown in [7]. At times when the time delay increases, i.e. the rate of change of the time delay is positive

$$
\dot{T}_{i}(t)=\frac{\mathrm{d} T_{i}(t)}{\mathrm{d} t}>0, \quad i=1,2,
$$

the communication line generates energy, thus is not passive. At decreasing time delay $\dot{T}_{i}(t)<0$ the communication line dissipates energy, hence is passive.

In order to preserve passivity the time varying gains

$$
f_{i}^{2}(t) \leq 1-\dot{T}_{i}(t), \quad i=1,2
$$




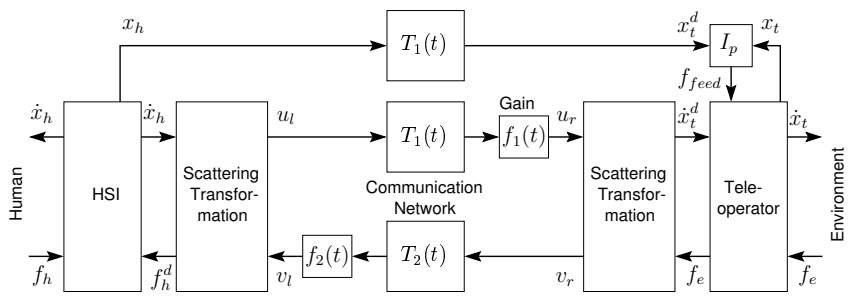

Fig. 3. Transparency orientated system architecture for time varying delay with time varying gains $f_{1}(t), f_{2}(t)$ and additional position feedforward.

are inserted shaping the energy output of the communication two-port depending on the rate of change of the transmission delay as shown in Fig. 2. For constant delay the gains calculate to $f_{1}=f_{2}=1$ recovering the results of [6].

\section{B. Transparency}

Ideal kinesthetic coupling expressed in the notion of transparency is achieved if the human operator feels directly connected to the remote environment. According to [5] this requires the positions and forces at the HSI and teleoperator to be equal

$$
x_{h}=x_{t} \wedge f_{h}=f_{e} .
$$

In the time varying delay case the passifying gains $f_{1}(t), f_{2}(t)$ transform the signals and thereby cause loss of transparency. Then a position drift between HSI and teleoperator may occur that is non-recoverable as velocity control cannot guarantee steady state error free position tracking. In order to overcome this deficiency an additional position feedforward is proposed in [8]. The position error

$$
e(t)=x_{h}\left(t-T_{1}(t)\right)-x_{t}(t)
$$

is weighted by feedforward gain $I_{p}$ acting as a proportional position controller with the (if necessary) saturated control output

$$
\begin{aligned}
& f_{\text {feed }}(t)=I_{p} \operatorname{sat}_{p}(e(t)) \\
& \text { with } \operatorname{sat}_{p}(e(t))=\left\{\begin{array}{ll}
e(t) \text { for }|e(t)| \leq p \\
p & \text { otherwise }
\end{array}, \quad p \geq 0,\right.
\end{aligned}
$$

see Fig. 3. The gain $I_{p}$ and the saturation $p$ are chosen so that the non-passivity of the position feedforward is compensated by the excess passivity of the teleoperator, for more details see [8].

\section{Communication Over Packet Switched NETWORKS}

We now consider a sampled data system for HSI and teleoperator and a dynamically routed multi-path packet switched network with time varying delay and packet loss, as e.g. the Internet, for communication. The communication line is decomposed into the packeting at the sender side, the packet transmission, and the packet processing algorithm at the receiver side, see Fig. 4. The goal of this section is to investigate the passivity and transparency of communication over packet switched networks with the main focus on typical packet processing algorithms.

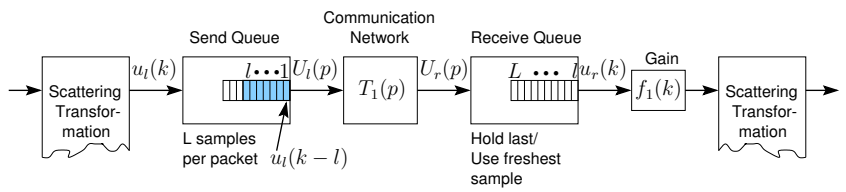

Fig. 4. Communication network with underlying packet nature and processing algorithms.

\section{A. Time Varying Delay and Packet Loss}

The sampled data system may be described as a continuous time system in which the variables are frozen for the sampling interval $T_{A}$. In what follows we indicate with $v(k)$ the value of the discrete variable $v(t)$ corresponding to the interval $t \in\left[k T_{A},(k+1) T_{A}\right]$. Discrete scattering transformation is applied at the sender and the receiver side, see [15], using the transformation equations given by (2) in discrete time now with the independent variable $k$. We assume that the sender as well as the receiver side operate with the same sampling interval $T_{A}$ and that the sampled data packets are sent in equal fixed time intervals over the network. For clarity the subsequent discussion on passivity refers to the forward path only, but equally applies to the backward path. In general a fixed number $L$ of consecutive samples of the wave variables $u_{l}(k)$ representing the output of the scattering transformation, are packed into one IPpacket $U_{l}(p)$ and put on the outgoing link.

During transmission over the network the IP-packet $U_{l}(p)$ experiences a load and routing policy dependent time varying link delay of $T_{1}(p)$. In a congested network IP-packets are discarded at the congested receive queues of intermittent routers resulting in packet loss. As a result the IP-packets may arrive at the receiving queue in time varying intervals, even in permutated order, or they may not arrive at all.

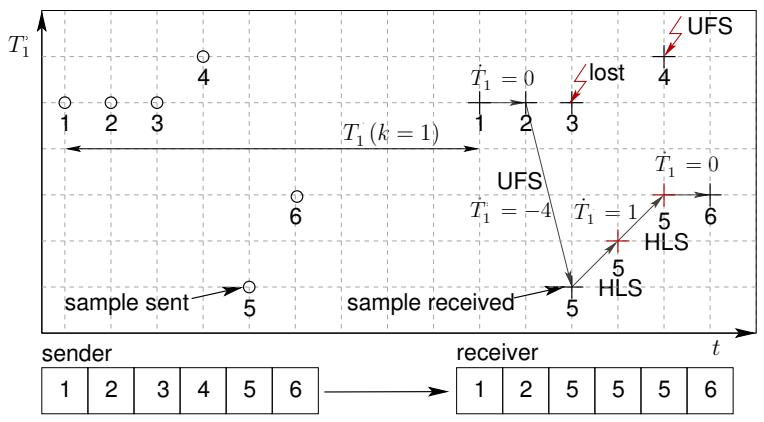

Fig. 5. Effect of processing algorithms UFS and HLS on the order of the received $(+)$ with respect to the sent $(\circ)$ packets and thereby on the rate of change of overall delay $\dot{T}_{1}$.

An example is given in Fig. 5, where the sent (o) and received $(+)$ packets are depicted with their arrival order. For simplicity we now consider data packets containing only one sample $u_{l}(k)$, thus $L=1$. The extension to data packets with more than one sample is straightforward. The $5^{\text {th }}$ packet in Fig. 5 arrives one time interval $T_{A}$ after the $2^{\text {nd }}$ packet, before the $4^{\text {th }}$ packet. The $3^{\text {rd }}$ packet is lost. The treatment of permutated order arrivals, late, and lost 


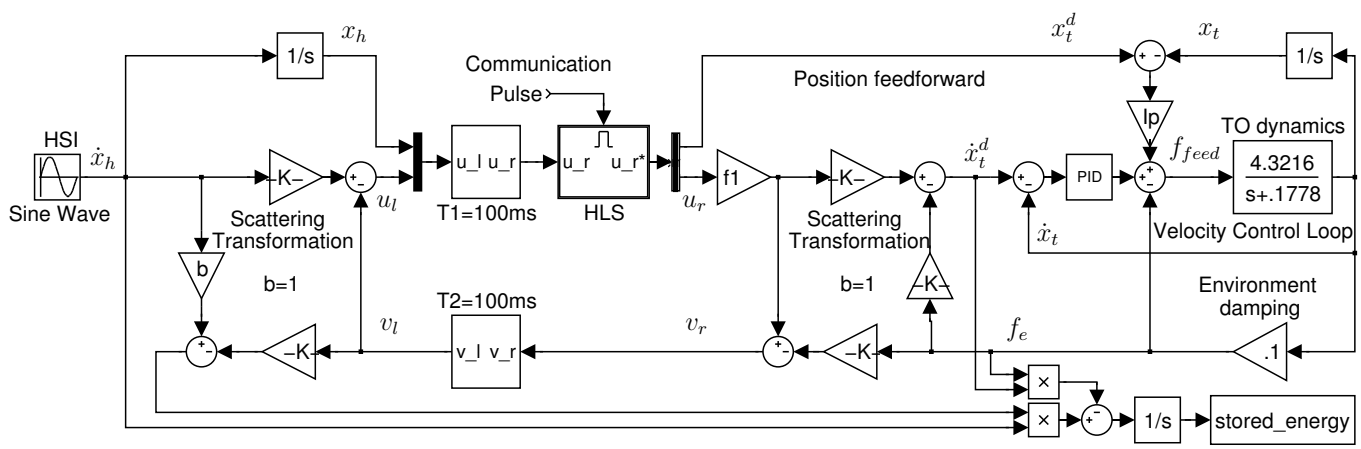

Fig. 6. Simulation structure for telepresence system with HLS and zeroing strategy for the velocity/ force and the position/velocity/force architecture.

packets is determined by the packet processing algorithm.

\section{B. Passivity of Use-Freshest-Sample}

In a real-time application, as the haptic telepresence system network is, delay plays a key role for performance and transparency. The goal is to minimize the delay introduced by the packet processing algorithm by of buffering. Therefore the Use-Freshest-Sample (UFS) algorithm without any buffering of data packets is applied here. Only the youngest packet is processed next, older packets, arriving too late are discarded. In Fig. 5 the $4^{\text {th }}$ packet is discarded in favor of the younger $5^{\text {th }}$ packet. At the cost of additional packet loss the effective communication delay of the packets is not increased by the UFS strategy. In correspondence with (4) non-increasing delay preserves the passivity of the communication two-port, hence the UFS algorithm is passive. Additional packet loss is induced by the UFS strategy.

\section{Non-Passivity of Hold-Last-Sample}

The processing induced packet loss as well as the network induced loss result in empty sampling instances at the receiver. The missing data can be estimated. One can imagine a whole class of estimation algorithms that use information of previous signal behavior. A very simple strategy belonging to this class of algorithms is the HoldLast-Sample (HLS) strategy. In case that there is no more data packet in the receive queue, the last sample is held until the next younger packet arrives, see Fig. 5. In terms of the time delay this means, that with every time step $T_{A}$ the overall delay increases by one time step $T_{A}$ corresponding to the rate of change $\dot{T}_{1}(k)=1$ indicating that the passivity condition (4) is violated. The non-passive behavior of the

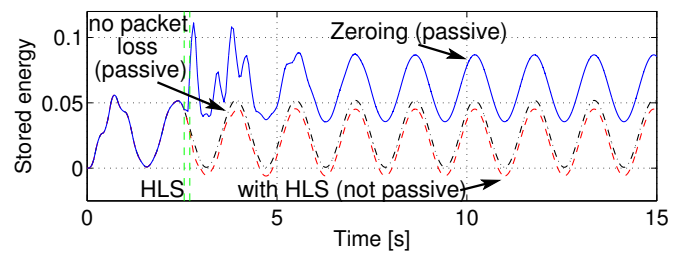

Fig. 7. Energy stored in Communication network without HLS, with HLS and with zeroing strategy.
HLS algorithm can be verified by the formal computation of the energy balance (3) of the communication two-port with HLS. Due to paper length constraints this proof is omitted.

For the HLS algorithm simulations are performed based on the simulation structure shown in Fig. 6 without the position feedforward extension, hence with the position feedforward control gain set to $I_{p}=0$. The time varying gain is set to $f_{1}=1$. The HSI is modeled as a pure velocity source providing a sinusoidal velocity signal with a frequency of $1 \mathrm{rad} / \mathrm{s}$. The teleoperator moves in free space with a small environment damping $b_{e}=0.1$. To show the effect of the HLS algorithm the delays in the forward and backward paths are kept constant $T_{1}=T_{2}=100 \mathrm{~ms}$. A single HLS is applied in the forward path at $t=2.5 \mathrm{~s}$ for a duration of $150 \mathrm{~ms}$. The energy generated in the communication twoport by this single HLS can be seen in Fig. 7, where negative values correspond to non-passivity; energy is computed from (3). The passivity condition (1) is violated. Without HLS passivity is preserved for constant time delay case, as expected.

\section{Passivity of the Zeroing Strategy}

If instead of holding the last sample a value of zero serves as output at empty sampling instances then no energy is generated at these instances. For the sketch of a proof a signal outage in the forward path between $t^{*}$ and $t$, hence $u_{r}(\tau)=0$ for $\tau \in\left[t^{*}, t\right]$ is considered. According to (3) the energy stored in the communication with zeroing computes to

$$
\int_{0}^{t} P_{i n, 0}(\tau) \mathrm{d} \tau=\frac{1}{2} \int_{0}^{t}\left(u_{l}^{2}+v_{r}^{2}-v_{l}^{2}\right) \mathrm{d} \tau-\frac{1}{2} \int_{0}^{t^{*}} u_{r}^{2} \mathrm{~d} \tau .
$$

As $t^{*}<t$ this expression is clearly larger than the expression in (3) showing that the output zeroing strategy strictly passifies the communication two-port. This relates to the continuous time computation of the time varying gain during HLS; with $\dot{T}_{1}=1$ applying (5) the time varying gain computes to $f_{1}=0$ recovering the zeroing strategy. In simulations the energy stored in the communication twoport takes strict positive values, see Fig. 7, satisfying the passivity condition (4).

The transparency though is decreased with the zeroing strategy. In the simulation according to Fig. 6 the gain is 
set to $f_{1}=0$ at $t=2.1 \mathrm{~s}$ for a duration of $500 \mathrm{~ms}$, hence a single signal zeroing occurs. For the standard velocity/force (VF) architecture a non-recoverable position error between HSI and teleoperator (TO) occurs, see Fig. 8, degrading transparency in terms of position tracking.

\section{E. Tracking Improvement by Position Feedforward}

The position/velocity/force architecture (PVF) has successfully been applied in the continuous time case improving the position tracking of HSI and teleoperator. Now we investigate the applicability in a packet switched network, where the position information $x_{h}$ is transmitted in the same data packet as the wave variable $u_{l}$. If a packet loss either due to congestion or the UFS packet processing algorithm occurs, the zeroing strategy is applied to the wave variable in order to preserve passivity. The HLS algorithm is applied to the position feedforward signal. Contrary to the foregoing discussion here the HLS is passive, as the feedforward controller output is designed by a saturation function guaranteeing passivity, see (7), and [8] for further details. As the simulation result in Fig. 8 shows, the position error induced by the zeroing strategy converges. The position/velocity/force architecture reveals good performance in terms of position tracking compared to the standard velocity/force architecture.

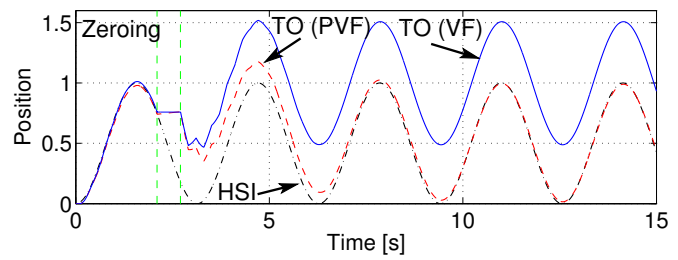

Fig. 8. Position tracking with zeroing strategy with (PVF) and without (VF) position feedforward.

In summary, the UFS algorithm is found to preserve passivity of the communication line in a packet switched network, whereas the HLS algorithm is not passive. Zeroing of the output values during signal outage strictly passifies the communication two-port but transparency in terms of position tracking is decreased. The position feedforward architecture with the zeroing strategy applied to the wave variable and HLS applied to the position signal preserves passivity, and increases transparency in terms of position tracking.

\section{Experimental System Architecture}

The experimental setup, see Fig. 9, consists of the HSI, a single degree of freedom force feedback paddle, refer to [3], and the teleoperator, a 7 DOF arm, see [12], each connected to a PC for control. The communication channel is represented by an emulated realtime communication network Berkeley Network Simulator (ns2) that is implemented on a third PC. IP packets containing haptic and administrative data as packet indices and time stamps are transmitted via IP socket connection on 100Mbps ethernet link between the HSI and the teleoperator via the network emulator PC. The

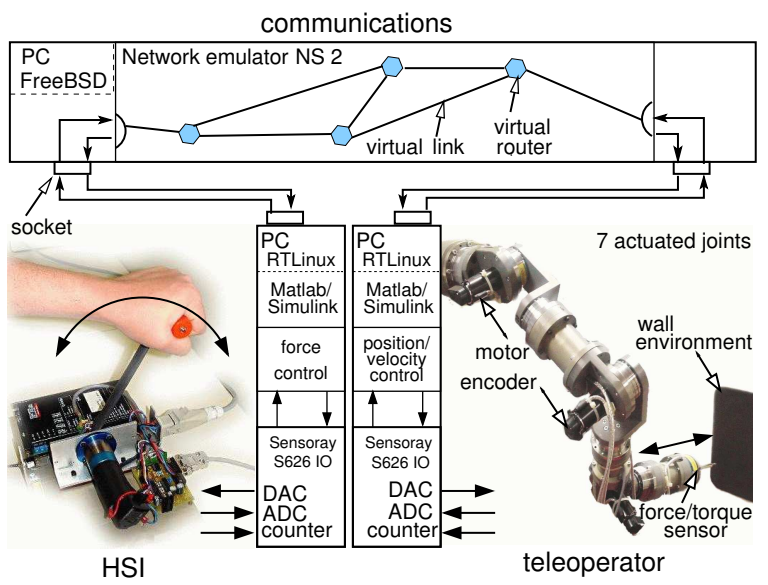

Fig. 9. Experimental system architecture.

HSI acts as the server, the teleoperator as the client. For more details on the experimental setup, see [4].

\section{EXPERIMENTAL RESULTS}

The following experiments are performed in order to investigate the transparency of the standard velocity/force (VF) architecture and the combined position/velocity/force (PVF) control approach when the telepresence system is operated over a packet switched network. Both strategies are compared in terms of position/force tracking, see the condition (6). Free space motion as well as contact with a wall is performed.

The angular position and velocity of the HSI (the paddle) are converted into linear motion variables (scaling factor $\frac{1}{3}$ $\mathrm{m} / \mathrm{rad}$ ) $x_{h}, \dot{x_{h}}$ and transmitted as set values for the Cartesian position and velocity to the teleoperator (7 DOF arm), see Fig. 9. The arm movement is limited to the Cartesian $y$ direction only. The arm first moves in free space, $0.16 \mathrm{~m}$ $(0.19 \mathrm{~m}$ for VF) from the initial position it contacts a wooden wall with a $3 \mathrm{~cm}$ thick sponge attached.

The VF architecture uses velocity-PI control on the slave side, the PVF applies velocity-P control with position feedforward. In order to have comparable results the position feedforward controller (PVF) is assigned the same value as the I-portion of the velocity control (PV). It turns out that the saturation of the output of the feedforward controller is not necessary for this setup. The force measured at the teleoperator end-effector is fed back as a set value for the force controlled paddle.

All controllers operate with a sampling interval of $T_{A}=$ $1 \mathrm{~ms}$. As in all experiments $L=20$ sample vectors are packed into one IP-packet an additional delay $T_{\text {pack }}=20 \mathrm{~ms}$ is introduced by packeting. The delay in the forward and the backward link is varied according to the pattern given by the upper plots in Fig. 10, so that the delay resulting from the packeting and the communication itself is $30 \mathrm{~ms} \leq T_{1}(t) \leq$ $100 \mathrm{~ms}$. Network induced loss is not considered. The UFSHLS packet processing algorithm is applied. Under the assumption that there is sufficient damping in the endsystems, 
the HLS-algorithm can be applied and the system remains stable throughout the experiment. The passifiying gain is chosen rather conservative $f_{1}(t)=f_{2}(t)=0.75$ for the duration of the experiment. The parameter of the scattering transformation is set to $b=1$.

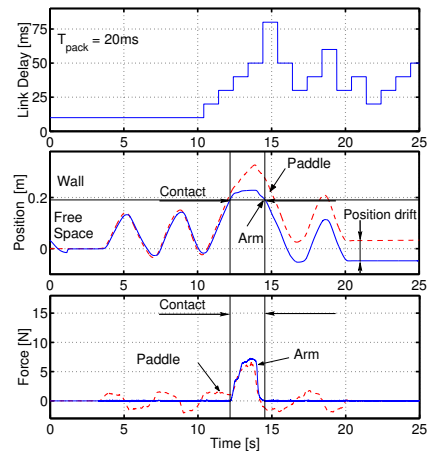

(a)

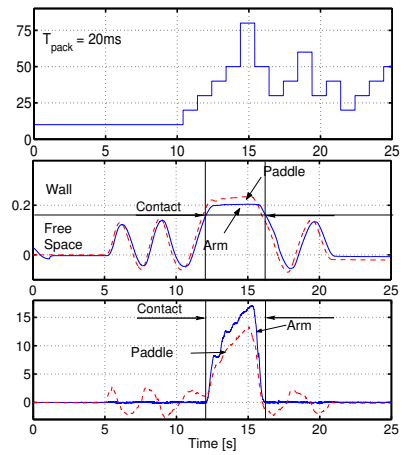

(b)
Fig. 10. Delay, position and force for standard VF architecture (a) and with additional position feedforward (PVF) (b)

Comparing the positions of the paddle and the arm for the VF and the PVF architecture reveals good tracking during free space motion, see middle plots in Fig. 10. In contact, however, the position of the paddle drifts away from the position of the arm resulting in a large steady state position error for the standard VF architecture. Adding position feedforward results in a very small position error. Main reason for not completely converging to zero is the non-compensated friction in the arm. Looking at the absolute values of the force in the contact situation for both architectures, see lower plots in Fig. 10, shows a large difference in the same environment, the force applied to the environment and displayed to the human in the PVF structure is about 2.5 times higher. Clearly, the PVF architecture is able to display higher stiffness than the VF. The stiffness is not included in the transparency condition given in (6) revealing the weakness of this measure; minimizing the tracking error of either position or forces independently is not sufficient for transparency.

In summary: By means of the position/velocity force architecture the operator is able to apply high forces to the environment, high stiffness is displayed and tracking of positions is superior compared to the VF architecture under time varying delay conditions as they occur in the Internet.

\section{CONCLUSIONS}

This paper investigates the existing stability conditions of haptic telepresence systems for the application in packet switched networks with time varying communication latencies (time delay) and packet loss, as e.g. the Internet. The impact of the packet processing protocol on the passivity and the performance is discussed. The Hold-LastSample algorithm is found to be not passive, whereas the Use-Freshest-Sample and the zeroing strategy is passive. The known position/velocity/force architecture is in- vestigated for their benefits in telepresence over packet switched networks. In experiments being the first steps towards a multimodal telepresence system over IP with rescue and other applications the system performance of the position/velocity/force architecture is compared with the standard velocity/force architecture, revealing good performance in terms of position tracking and displaying high stiffness.

\section{Acknowledgments}

The authors acknowledge the contributions by B. Stanczyk during the experiments performed with the teleoperator arm. The detailed comments by the anonymous reviewers are highly appreciated.

\section{REFERENCES}

[1] M. Buss and G. Schmidt, "Control Problems in Multi-Modal Telepresence Systems," in Advances in Control: Highlights of the 5th European Control Conference ECC'99 in Karlsruhe, Germany (P. Frank, ed.), pp. 65-101, Springer, 1999.

[2] K. Goldberg, S. Gentner, C., and J. Wiegley, "The Mercury Project: A Feasibility Study for Internet Robots," UC Berceley and University of Southern California, 1994.

[3] H. Baier and M. Buss and G. Schmidt, "Control Mode Switching for Teledrilling Based on a Hybrid System Model," in Proceedings of the IEEE/ASME International Conference on Advanced Intelligent Mechatronics AIM'97, 1997, Tokyo, Japan, Paper No. 50.

[4] Hirche, S., Stanczyk, B., and Buss, M. "Transparent Exploration of Remote Environments by Internet Telepresence," In Proceedings of the International Workshop on High-Fidelity Telepresence and Teleaction, jointly with the conference HUMANOIDS, (Munich, Germany), 2003.

[5] Y. Yokokohji and T. Yoshikawa, "Bilateral Control of Master-Slave Manipulators for Ideal Kinesthetic Coupling Formulation and Experiment," IEEE Transactions on Robotics and Automation, vol. 10, pp. 605-619, October 1994.

[6] R. Anderson and M. Spong, "Bilateral Control of Teleoperators with Time Delay," IEEE Transaction on Automatic Control, vol. 34, pp. 494501, 1989.

[7] N. Chopra, R. Lozano and M. Spong, "Passivation of Force Reflecting Bilateral Teleoperators with Time Varying Delay," in Proceedings of the 8. Mechatronics Forum, (Enschede, Netherlands), 2002, pp. 954962.

[8] N. Chopra, M. W. Spong, S. Hirche, and M. Buss, "Bilateral Teleoperation over Internet: the Time Varying Delay Problem," in Proceedings of the American Control Conference, (Denver, CO), 2003, pp. 156-160.

[9] K. Kosuge and H. Murayama and K. Takeo, "Bilateral Feedback Control of Telemanipulators via Computer Network," in Proceedings of the IEEE/RSJ International Conference on Intelligent Robots and Systems IROS, 1996, Osaka, Japan, pp. 1380-1385.

[10] J. Nilsson, "Real-time Control Systems with Delays," PhD thesis , Lund Institute of Tecnology, 1998.

[11] Q. Ling and M.D. Lemmon, "Robust Performance of Soft Real-time Networked Control Systems with Data Dropout," in Proceedings of the 41nd IEEE Conference on Decision and Control CDC'02, 2002, Las Vegas, US, pp. 1225-1230.

[12] B. Stanczyk, "Teleoperated Exploration and Manipulation of Hazardous Environments with Dual Arm Robot/ Avatar," Technical Report TR-RS-2003-01-3, Control Systems Group, Technical University of Berlin, 2003.

[13] S. Hirche and M. Buss, "Study of Teleoperation using Realtime Communication Network Emulation," in Proceedings of the International Conference on Advanced Intelligent Mechatronics, (Kobe, Japan), 2003, pp. 586-591.

[14] G. Niemeyer and J.-J. Slotine, "Stable Adaptive Teleoperation," IEEE Journal of Oceanic Engineering, vol. 16, pp. 152-162, January 1991.

[15] Stramigioli, S., van der Schaft, A., Maschke, B. and Melchiorri, C., "Geometric Scattering in Robotic Telemanipulation." IEEE Transactions on Robotics and Automation 20, vol. 18, pp. 588-596, August 2002. 